\title{
ANÁLISIS DE LA HETEROGENEIDAD DE LA DEMANDA SOCIAL POR POLÍTICAS DE MITIGACIÓN DE LA CONTAMINACIÓN DIFUSA EN AGRICULTURA
}

\author{
Francisco Alcon a*, José A. Zabala ${ }^{\text {a }}$, José M. Martínez-Paz \\ a Departamento de Economía de la Empresa. Universidad Politécnica de Cartagena. (Cartagena, \\ francisco.alcon@upct.es, joseangel.zabala@upct.es) \\ ${ }^{b}$ Departamento de Economía Aplicada. Universidad de Murcia. (Murcia,jimpaz@um.es)
}

\begin{abstract}
Resumen
Este trabajo tiene como objetivo valorar la demanda social por medidas de gestión agrícola tendentes a mitigar la contaminación difusa, así como analizar la heterogeneidad entre la población de las preferencias. Se utiliza como caso de estudio las medidas propuestas por la Ley 1/2018, de 7 de febrero, para garantizar la sostenibilidad ambiental en el entorno del Mar Menor, destinadas a mitigar la contaminación por nitratos en la zona del Campo de Cartagena (Región de Murcia, España). El método empleado ha sido un experimento de elección, que ha permitido evaluar la disposición al pago por un total de cuatro medidas agrícolas. La heterogeneidad se ha analizado en base a los resultados del modelo logit mixto de clases latentes estimado.

Los resultados revelan la existencia de heterogeneidad en la demanda de medidas, identificando dos clases latentes en base a su compromiso ambiental y su opinión sobre los factores causantes de la degradación del Mar Menor. Los beneficios socioeconómicos derivados de la aplicación de las medidas superan los costes que deben asumir los agricultores para su implementación, que alcanzan los $1.600 € / \mathrm{ha} /$ año. Los resultados proporcionan información a los gestores públicos para el establecimiento de estrategias de mitigación de la contaminación por nitratos agrícolas basadas en los beneficios de las medidas.
\end{abstract}

Palabras clave: Contraservicios ecosistémicos, Experimentos de elección, Externalidades, Preferencias sociales.

\section{Introducción y objetivos}

La degradación de los ecosistemas acuáticos causada por la contaminación por nutrientes agrícolas es un problema medioambiental que puede verse agravado en los próximos años debido al incremento en la demanda de alimentos, con la consecuente intensificación agrícola, y los efectos negativos del cambio climático [WWAP (2018)]. El reto es entonces la gestión eficiente los impactos negativos procedentes de la escorrentía y lixiviación de los nutrientes agrícolas. Para ello, la implementación de medidas de gestión en los cultivos es una de las vías para conseguir reducir estos impactos.

Las medidas de gestión agrícola incluyen en muchas ocasiones una modificación de las actuales prácticas de cultivo, lo que supone asimismo cambios en la provisión de servicios ecosistémicos, más allá de la mitigación del contraservicio de la contaminación por nutrientes [Alcon et al. (2021)]. Es por ello que las implicaciones de la adopción de prácticas de cultivo superan los límites del sector agrícola y transciende a toda la sociedad. La participación pública se convierte, por tanto, en una herramienta necesaria para dar soporte a las acciones políticas que potencien medidas agrícolas socialmente aceptadas.

En este contexto, el presente trabajo tiene como objetivo valorar la demanda social por medidas agrícolas para mitigar la contaminación difusa de la agricultura y los beneficios ambientales que, derivados de su implementación, se consiguen en los ecosistemas acuáticos adyacentes. Además, la heterogeneidad en las preferencias sociales ha sido analizada.

\section{Metodología}

\subsection{Caso de estudio}

Se ha utilizado como caso de estudio la comarca del Campo de Cartagena (Región de Murcia, España), en la que coexisten una importante actividad agrícola intensiva y el Mar Menor, ecosistema acuático que recibe los impactos negativos de la agricultura. El Campo de Cartagena posee un total de 44.000 ha de cultivo en régimen de regadío intensivo, produciendo frutas y hortalizas de alto valor añadido, con una orientación eminentemente exportadora. Esta comarca vierte sus aguas, tanto superficiales como subterráneas, al Mar Menor, la mayor laguna costera de agua salada del Mediterráneo con hábitats singulares y con múltiples figuras de protección internacionales (ej. Natura 2000, Humedal Ramsar).

Los episodios de eutrofización que viene sufriendo el Mar Menor, cada vez más frecuentes y evidentes (como la explosión de fitoplancton del verano de 2016) hace que su situación ecológica se haya trasladado 
a la opinión pública [Perni et al. (2020)], poniendo el foco de atención sobre el sector agrícola, como uno de los causantes de su degradación. El fuerte debate sociopolítico concluyó con la aprobación de la Ley 1/2018, de 7 de febrero de 2018, de medidas urgentes para asegurar la sostenibilidad ambiental en el entorno del Mar Menor. Esta ley, de elevada controversia social que ha llevado a que sufra sucesivas modificaciones, establece finalmente las siguientes medidas agrícolas con el propósito de reducir la escorrentía y lixiviación de nutrientes de la comarca del Campo de Cartagena hacia el Mar Menor:

- Prohibición de cultivos de hortalizas a menos de $100 \mathrm{~m}$ de la costa ("Cultivo > $100 \mathrm{~m}$ de costa").

- Instalación de un sistema para reducir el contenido en nitratos del agua obtenida de las plantas desalobradoras antes de su uso en cultivos y su entrada a las aguas subterráneas y/o al Mar Menor ("Plantas desnitrificadoras").

- Establecimiento de setos alrededor del perímetro de las fincas ("Seto perimetral”).

- Cumplimiento de un código de buenas prácticas agrícolas (BPA) basado en el uso eficiente de fertilizantes y agua de riego, de acuerdo con la Directiva de Nitratos ("Código BPA").

\subsection{Experimentos de elección}

El método empleado para la valoración de la demanda social ha sido el de los experimentos de elección. El Cuadro 1 recoge los atributos y niveles del experimento, formados por las medidas agrícolas recogidas en la Ley 1/2018, de 7 de febrero, así como los posibles niveles de mejora en la calidad del agua del Mar Menor. Estos niveles han sido definidos en base a indicadores cualitativos de calidad biológica en el ecosistema, tales como la reducción en la presencia de algas y fitoplancton y la mejora en la diversidad y abundancia de hábitats bentónicos. Como vehículo de pago se ha optado por una reasignación de los impuestos que actualmente paga cada hogar para destinar una parte a financiar la implementación de las citadas medidas agrícolas durante los próximos cinco años.

Cuadro 1. Atributos y niveles del experimento de elección

\begin{tabular}{|c|c|c|}
\hline Atributo & Definición & Niveles \\
\hline $\begin{array}{l}\text { Mejora en la } \\
\text { calidad del agua }\end{array}$ & $\begin{array}{l}\text { Mejora de la calidad del agua (presencia } \\
\text { de algas y fitoplancton | hábitats } \\
\text { bentónicos) en el Mar Menor }\end{array}$ & $\begin{array}{l}\text { No mejora (SQ) } \\
\text { Mejora moderada (referencia) } \\
\text { Mejora elevada }\end{array}$ \\
\hline $\begin{array}{l}\text { Medidas } \\
\text { agrícolas }\end{array}$ & $\begin{array}{l}\text { Medidas agrícolas establecidas por la Ley } \\
1 / 2018 \text { para asegurar la sostenibilidad } \\
\text { medioambiental del Mar Menor }\end{array}$ & $\begin{array}{l}\text { Ninguna medida (SQ) } \\
\text { Cultivo }>100 \mathrm{~m} \text { de costa (referencia) } \\
\text { Plantas desnitrificadoras } \\
\text { Seto perimetral } \\
\text { Código BPA }\end{array}$ \\
\hline $\begin{array}{l}\text { Coste } \\
\text { (€/hogar/año) }\end{array}$ & $\begin{array}{l}\text { Impuestos reasignados para apoyar las } \\
\text { medidas agrícolas durante los próximos } \\
\text { cinco años }\end{array}$ & $10|20| 30 \mid 40$ \\
\hline
\end{tabular}

Fuente: Elaboración propia

Los niveles han sido combinados en un diseño S-eficiente, dando lugar a un total de 16 conjuntos de elección agrupados en 4 bloques, asignados aleatoriamente a cada encuestado. Cada conjunto de elección estaba compuesto de 3 alternativas, una de las cuales representaba la situación actual, o status quo (SQ).

Las elecciones de los encuestados fueron modelizadas mediante un modelo logit mixto de clases latentes. Este modelo permite analizar la heterogeneidad en las preferencias asumiendo que los individuos pueden ser clasificados en distintas clases con preferencias similares y que, a su vez, las utilidades marginales de cada atributo para cada clase pueden variar siguiendo una determinada distribución de probabilidad. Se ha asumido que los coeficientes siguen una distribución normal, para el caso de los atributos no monetarios, y una log-normal negativa para el atributo coste. Asimismo, los factores sociodemográficos y actitudinales que determinan la probabilidad de pertenecer a cada clase también son analizados. El número de clases óptimo ha sido seleccionado según el criterio de información de Akaike.

El experimento de elección fue desarrollado a través de encuestas personales durante el verano de 2019. La población objetivo correspondía al conjunto de la Región de Murcia (543.800 hogares), contando con una muestra final de 576 hogares.

\section{Resultados}

El Cuadro 2 recoge los resultados de la modelización de las elecciones, después de haber eliminado las respuestas protesta (88 encuestados $\sim 15,28 \%$ de la muestra). Estos resultados muestran la existencia de 
dos clases latentes diferenciadas según las preferencias individuales. La clase 1, que incluye a dos tercios de los encuestados, se muestra a favor de implementar todas las medidas agrícolas propuestas, y prefieren la implementación de plantas desnitrificadoras, setos perimetrales y el código BPA antes que prohibir los cultivos a menos de $100 \mathrm{~m}$ de la costa (referencia). Por el contrario, las preferencias de los individuos de la clase 2 , que incluye a un tercio de los encuestados, indican que se muestran contrarios a aplicar este tipo de medidas agrícolas, percibiendo desutilidad con su implementación. Como era de esperar, la mejora elevada en la calidad del agua del Mar Menor presenta un coeficiente positivo y significativo para ambas clases, demostrando la utilidad que la población obtiene de su incremento. No obstante, se observa que los individuos pertenecientes a la clase 2 obtienen mayores niveles de utilidad de la mejora en la calidad del agua. Ello está en consonancia con la desutilidad percibida por la situación actual (SQ), cuyo impacto negativo también es superior en el caso de la clase 2. Del análisis de la asignación de clases, se puede comprobar que los individuos pertenecientes a la clase 2 son aquellos con un mayor compromiso ambiental y quienes otorgan a la agricultura una mayor responsabilidad como actividad causante de la actual degradación del Mar Menor.

Cuadro 2. Función de utilidad subyacente. Modelo de elección logit mixto de clases latentes.

\begin{tabular}{lcccc}
\hline & \multicolumn{2}{c}{ Clase 1 } & \multicolumn{2}{c}{ Clase 2 } \\
& Coef. (Err. Est.) & Coef. (Err. Est.) & Coef. (Err. Est.) & Coef. (Err. Est.) \\
\hline SQ & Media & Desv. Est. & Media & Desv. Est. \\
Mejora elevada & $-4,08(0,24) * * *$ & $-1,91(0,50) * * *$ & $-8,37(1,73) * * *$ & $3,34(1,28) * * *$ \\
Plantas desnitrificación & $0,24(0,14) *$ & $-0,73(0,31) * *$ & $4,83(0,59) * * *$ & $1,91(1,03) *$ \\
Setos perimetrales & $0,84(0,18) * * *$ & $0,91(0,58)$ & $-5,25(0,93) * * *$ & $6,52(1,77) * * *$ \\
Código BPA & $0,76(0,15) * * *$ & $-0,25(0,66)$ & $-3,56(1,38) * * *$ & $5,85(0,66) * * *$ \\
Coste & $-0,09(0,01) * * *$ & $0,18(0,04) * * *$ & & \\
Asignación de clases & & & & \\
Probabilidad & 0,67 & & & \\
Constante & $5,30(1,48) * * *$ & & & \\
Usuario & $0,04(0,37)$ & & & \\
Compromiso ambiental & $-0,56(0,24) * *$ & & & \\
Edad & $-0,01(0,01)$ & & & \\
Educación superior & $-0,41(0,39)$ & & & \\
Impact-Agria & $-0,36(0,16) * *$ & & & \\
\hline Na: Nivi & & & & \\
\hline
\end{tabular}

Nota: Nivel de significatividad al $* 10 \%, * * 5 \%, * * * 1 \%$.

a Percepción de los encuestados acera del impacto de la agricultura sobre el estado ecológico del Mar Menor (medido mediante escala Likert de 5 puntos)

Fuente: Elaboración propia

Derivado de las preferencias del modelo de elección, el Cuadro 3 muestra la disposición a pagar (DAP) por las medidas agrícolas y la mejora de la calidad del agua del Mar Menor. El análisis de esto resultados pone de manifiesto que, pese a que quienes pertenecen a la clase 2 no se muestran favorables a la implementación de las medidas comentadas, el valor promedio para el conjunto de la sociedad es positivo. Ello da soporte a la implementación de todas ellas, algo especialmente respaldado por los valores negativos de DAP que refleja la situación actual, así como los impactos positivos que se producen con la mejora de la calidad del agua.

Cuadro 3. DAP marginal por la mejora elevada en la calidad del agua y las medidas agrícolas (€/hogar/año)

\begin{tabular}{lccc}
\hline & Clase 1 & Clase 2 & Media ponderada \\
\hline SQ & $-80,98$ & $-154,88$ & $-105,37$ \\
Mejora de calidad elevada & 6,48 & 79,70 & 30,64 \\
Plantas desnitrificadoras & 18,19 & $-30,85$ & 2,01 \\
Setos perimetrales & 15,74 & $-11,08$ & 6,89 \\
Código BPA & 14,07 & $-11,34$ & 5,69 \\
\hline
\end{tabular}

Fuente: Elaboración propia

Con esta información y la correspondiente a los costes de implementación de las medidas para el agricultor, se han definido una serie de escenarios para su comparación en términos de coste-beneficio (Cuadro 4). De este modo se observa que, independientemente del nivel de mejora en la calidad del agua conseguido, el escenario 1 que implica eliminar los cultivos a menos de $100 \mathrm{~m}$ de la costa, es el que mejor ratio B/C presenta, a pesar de no ser el que más beneficios proporciona a la sociedad. 
Cuadro 4. Costes y beneficios de los escenarios de gestión agrícola para mitigar la contaminación por nutrientes (€/ha/año)

\begin{tabular}{|c|c|c|c|c|c|c|c|}
\hline & Medida agrícola & $\mathrm{CAE}^{\mathrm{a}}$ & $\begin{array}{l}\text { Mejora en la } \\
\text { calidad del } \\
\text { agua }^{\mathrm{b}}\end{array}$ & Clase 1 & $\begin{array}{l}\text { BAE c } \\
\text { Clase } 2\end{array}$ & Promedio & Ratio B/C \\
\hline \multirow[t]{2}{*}{ Escenario 1} & Cultivo $>100 \mathrm{~m} \mathrm{de}$ & 12,23 & (1a) Moderado & 1.001 & 1.914 & 1.302 & 106,48 \\
\hline & costa & & (1b) Alto & 1.081 & 2.899 & 1.681 & 137,45 \\
\hline \multirow[t]{2}{*}{ Escenario 2} & Plantas & 70,00 & (2a) Moderado & 1.226 & 1.533 & 1.327 & 18,96 \\
\hline & desnitrificadoras & & (2b) Alto & 1.306 & 2.517 & 1.706 & 24,37 \\
\hline \multirow[t]{2}{*}{ Escenario 3} & Setos perimetrales & 325,81 & (3a) Moderado & 1.370 & 1.637 & 1.458 & 4,47 \\
\hline & Código BPA & & (3b) Alto & 1.449 & 2.622 & 1.836 & 5,64 \\
\hline \multirow[t]{2}{*}{ Escenario 4} & $\begin{array}{l}\text { Plantas } \\
\text { desnitrificadoras }\end{array}$ & 168,14 & (4a) Moderado & 1.420 & 1.396 & 1.412 & 8,40 \\
\hline & Setos perimetrales & & (4b) Alto & 1.500 & 2.381 & 1.791 & 10,65 \\
\hline
\end{tabular}

Nota: CAE and BAE estimados para un período de 5 años y usando una tasa de descuento social del 3,50\%, considerando 543.800 hogares y 44.000 ha de cultivo en regadío.

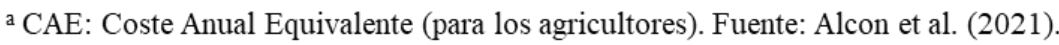

${ }^{b}$ Ambos niveles de mejora en la calidad del agua pueden ser alcanzados con cualquier escenario.

cBAE: Beneficio Anual Equivalente.

Fuente: Elaboración propia

\section{Conclusiones}

La implementación de medidas agrícolas para la mitigación de la contaminación difusa requiere de acciones agrícolas y del apoyo de la sociedad. En este sentido, el presente trabajo ha puesto de manifiesto la existencia de una demanda social por medidas agrícolas tendentes a mitigar la contaminación por nitratos y mejorar así la calidad del agua de ecosistemas costeros adyacentes. No obstante, ello ha servido también para corroborar la existencia de opiniones contrariadas acerca del papel que estas medidas deben tener.

Con todo ello, los resultados pretenden servir de apoyo a la implementación eficiente de la política, de modo que se pueden considerar las siguientes recomendaciones: (1) se deben implementar las medidas agrícolas propuestas, dados los beneficios que brindan; (2) los beneficios socioeconómicos derivados de las medidas podrían aplicarse para financiar un programa de ayuda a los agricultores, que garantizase la aplicación de las medidas (políticas basadas en incentivos); (3) debe garantizarse que las medidas consigan alcanzar una elevada calidad del agua en los ecosistemas adyacentes, dados los elevados beneficios que genera.

\section{Agradecimientos}

Este trabajo se ha realizado en el marco del proyecto 20912/PI/18 financiado por la "Fundación SénecaAgencia de Ciencia y Tecnología de la Región de Murcia”. José A. Zabala agradece la ayuda recibida del MEFP (FPU16/03473).

\section{Bibliografía}

Alcon, F., de-Miguel, M.D. y Martínez-Paz, J.M. (2021). "Assessment of real and perceived costeffectiveness to inform agricultural diffuse pollution mitigation policies". Land Use Policy, 107, 104561.

Perni, A., Barreiro-Hurlé, J. y Martínez-Paz, J. M. (2020). "When policy implementation failures affect public preferences for environmental goods: Implications for economic analysis in the European water policy". Ecological Economics, 169: 106523.

WWAP (United Nations World Water Assessment Programme). (2018). The United Nations World Water Development Report 2018: Nature-Based Solutions for Water. UNESCO, Paris. 